\title{
Structural and Functional Analysis of a New Upstream Promoter of the Human FAT/CD36 Gene
}

\author{
Chikako Kuriki, ${ }^{a}$ Takao Tanaka, ${ }^{b}$ Yuka Fukui, ${ }^{a, c}$ Osamu SAto,${ }^{a, c}$ and Kiyoto Motojima ${ }^{*, a, c}$ \\ ${ }^{a}$ Department of Biochemistry, School of Pharmaceutical Sciences, Toho University; 2-2-1 Miyama, Funabashi, Chiba \\ 274-8510, Japan: ${ }^{b}$ Third Division, Department of Internal Medicine, Osaka Medical College; Takatsuki, Osaka \\ 569-8686, Japan: and ${ }^{c}$ Department of Biochemistry, Meiji Pharmaceutical University; 2-522-1 Noshio, Kiyose, Tokyo \\ 204-8588, Japan. Received August 22, 2002; accepted September 3, 2002
}

FAT/CD36 is involved in various processes including uptake of fatty acid into the heart and of oxidized low density lipoprotein (LDL) into macrophages. Expression of the FAT/CD36 gene is regulated in a tissue-specific manner, and loss or inadequately regulated expression of FAT/CD36 is thought to be one of the causes of some diseases such as cardiomyopathy and atherosclerosis. We recently found that the mouse and human FAT/CD36 genes have two independent promoters. To elucidate the physiological significance of the two promoters, we characterized the peroxisome proliferator-activated receptor ligand-responsive new promoter that is located $14 \mathrm{~kb}$ upstream of the previously reported promoter of the human gene. We found several SNPs in this region some of which were found only when analyzing DNA samples from the patients lacking FAT/CD36 totally or in a celltype-specific manner. However, we could not detect any negative effect of these SNPs on the transcription by transient transfection analysis, suggesting that the identified SNPs alone are not directly linked to low transcriptional activities.

Key words FAT; CD36; PPAR; promoter; SNP

FAT/CD36 is an $88 \mathrm{kDa}$ membrane glycoprotein expressed on the surface of several cell types playing diverse functions including uptake of long chain fatty acids (LCFA) and oxidized low density lipoprotein (LDL) ${ }^{1,2)}$ Recent studies have revealed that uptake of these molecules is not a simple diffusion process across the plasma membrane but is mediated by several membrane proteins (reviewed in ref. 3) and regulated at the transcriptional, ${ }^{4,5}$ translational ${ }^{6)}$ and post-translational stages. ${ }^{7}$ These findings suggest that uptake of LCFA and oxidized LDL are important steps in lipid homeostasis at the whole body level and should be regulated precisely in coordination with intracellular metabolism. Thus, abnormalities in expression and regulation of the transport proteins may contribute to the onset of several diseases and compounds that modulate their expression and activities are expected to become useful as therapeutic drugs.

In addition to the studies using the FAT/CD36-deficient rodents, ${ }^{8,9)}$ the demonstration that a defect in human myocardial LCFA uptake is caused by FAT/CD36 mutations ${ }^{10}$ indicated that FAT/CD36 is actually a fatty acid transporter in adipocytes and muscle cells, although the mutations identified to date can explain only the total deficiency but not cell type-specific deficiency of FAT/CD36; that is, loss of the protein in platelets and normal expression in monocytes. ${ }^{11)}$

We have reported that the mouse and human FAT/CD36 genes have two independent promoters. ${ }^{12)}$ Northern blot and functional analysis of the mouse promoters suggested that only the newly identified upstream promoter indirectly responds to the peroxisome proliferator-activated receptor (PPAR) ligands. RT-PCR analysis of the $5^{\prime}$-end of the human mRNA also suggested that only the upstream of the human gene responds to the PPAR ligands. ${ }^{12}$ ) To further examine whether the upstream promoter of the human FAT/CD36 gene (summarized in Fig. 1) is independently active as in the mouse gene, we carried out structural and functional analysis of the promoter sequences of the DNA samples from the FAT/CD36 deficient patients.

\section{MATERIALS AND METHODS}

Materials Dual-Luciferase Reporter Assay System and pGL reporter plasmids were purchased from Promega (Wisconsin, U.S.A.). KOD polymerase and restriction enzymes were obtained from Toyobo (Osaka, Japan).

Genomic DNA Amplification and Sequencing The genomic DNAs were isolated from 15 blood samples from type I and type II FAT/CD36 deficient patients and normal subjects. The genomic DNAs were amplified by PCR using a set of specific primers designed according to the distal promoter sequence ${ }^{12)}$ (GenBank accession no. AF434768) and the products were directly sequenced. The primer sequences were 5'-GGCTAGGGACCAGCTCGAGAAT (left) and 5'GATGAAAGAGCACAAGGCCACA (right). SequiTherm EXCEL II Long-Read ${ }^{\mathrm{TM}}$ DNA sequencing kit (Epicentre Technologies, Wisconsin, U.S.A.) was used for the reaction and the fragments were analyzed using a DNA sequencer (Model 4000, LI-COR).

Cloning of Upstream Promoter Sequences The upstream promoter sequences of the FAT/CD36 gene of 8 patients and 3 normal subjects were amplified by PCR using the left primer (5'-GAGAGCTCTGGGGGTCTCTGAACCC) and the right primer (5'-GCGCTAGCTAGTTGGGAAGACCAGGG) designed from the previously determined sequence. ${ }^{12)}$ The reaction products were treated with the restriction enzymes, Sac I and Nhe I and the $1.5 \mathrm{~kb}$ fragments were cloned into the Sac I-Nhe I site of pGL3-promoter vector (Promega).

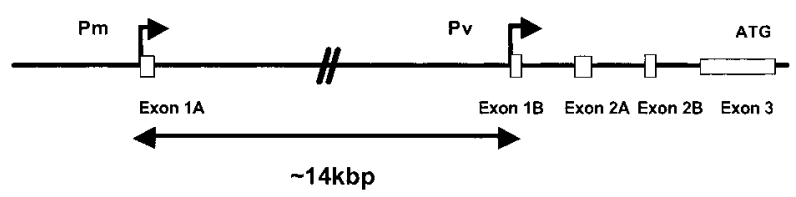

Fig. 1. Summary of the Dual Promoter Region of the Human FAT/CD36 Gene 
Cell Culture and DNA Transfection Human hepatoblastoma HepG2 cells were cultured in standard medium (Dulbecco's modified Eagle's medium containing $25 \mathrm{~mm}$ glucose, $2 \mathrm{~mm}$ glutamine, and $10 \%$ bovine serum). Transfection of HepG2 cells was performed in 24-well plates with SuperFect ${ }^{\mathrm{TM}}$ (Qiagen). The transfected cells were cultured in growth media for $24 \mathrm{~h}$ and the luciferase activities were measured using the Dual-Luciferase Assay System (Promega) according to the manufacturer's protocol as described previously. ${ }^{12)}$

\section{RESULTS AND DISCUSSION}

FAT/CD36 deficiency is heterogeneous in phenotype and can be grouped into two classes (see Fig. 2). Type I is a total loss of the protein in platelet and monocyte membranes. This is caused by mutations in the coding exons of the FAT/CD36 gene or at a splice junction as recently reported. ${ }^{10)}$ In contrast, type II is a cell type-specific deficiency, i.e. a loss of the protein in platelets but normal expression in monocytes. ${ }^{11)}$ This unique deficiency cannot be explained by mutations in the coding region. Accordingly, we are interested in the possibility that the cell type-specific deficiency of FAT/CD36 is caused by mutations in one of the two promoters of the gene. Our observation that both promoters are active in monocytic THP-1 cells ${ }^{12)}$ suggested that the cells leading to platelets use only one of the two promoters and mutations in the promoter region would change the expression levels of FAT/CD36 in the platelets.

To examine the possibility that the platelet-specific deficiency of FAT/CD36 is caused by mutations in the distal promoter sequence because no mutations have been found to date in the published downstream promoter sequence, ${ }^{11,13)}$ we analyzed 15 blood samples from type I, and type II deficient patients and normal subjects. The genomic DNAs were amplified by PCR and the products were directly sequenced. The results are summarized in Fig. 3. In the distal promoter region from position -1 to -1000 relative to the transcriptional start site, we found 5 SNPs. The SNPs at $-705,-729$ and -730 were found in all three groups, and thus do not affect the transcriptional activities of the promoter. The SNP at -14 was found as a homozygous form A/A in 7 of 8 patients with type I deficiency, and in none of 3 type I patients or 3 normal subjects. The SNP at -593 was found as a homozygous $\mathrm{G} / \mathrm{G}$ or a heterozygous $\mathrm{G} / \mathrm{A}$ form in 10 of 11 patients with type I or type II deficiency, and in none of the normal subjects. The sequence published by the Human Genome Project coincides with our wild-type sequence. These results suggested that the SNPs in the distal promoter region, especially at -14 , have some effects on the transcriptional activity of the gene.

To directly examine the correlation between the SNPs found in the distal promoter region and transcriptional activities of the variant promoters, we cloned the PCR-amplified $1.5 \mathrm{~kb}$ fragments into a reporter plasmid and measured their activities by reporter assay. For this purpose, we used HepG2 cells because no platelet cell line could be obtained and HepG2 cells use only the distal promoter of the FAT/CD36 gene $^{12)}$ as cells leading to platelets.

The luciferase activities of individual reporter plasmids in HepG2 cells are summarized in Fig. 4. All promoters were

\begin{tabular}{|c|c|c|c|c|}
\hline \multicolumn{2}{|c|}{} & Platelet & Monocyte & Sample No. \\
\hline \multicolumn{2}{|c|}{ Type I } & - & - & $11,12,13$ \\
\hline \multirow{3}{*}{ Type II } & \multirow{2}{*}{ (1) } & - & $+\sim++$ & $\begin{array}{c}1,2,3,4, \\
5,7,8,9\end{array}$ \\
\cline { 2 - 5 } & \multirow{2}{*}{ (2) } & + & $+\sim++$ & 6 \\
\hline \multirow{2}{*}{ Wild Type } & ++ & ++ & $21,22,23$ \\
\hline
\end{tabular}

Fig. 2. Summary of the Phenotypes of FAT/CD36 Deficiencies and the DNA Samples Analyzed in This Study

The symbols,-+ and ++ represent the expression levels of FAT/CD36 protein. The levels were analyzed by flow cytometry after staining with monoclonal antiFAT/C36 antibody (see refs. 12 and 13 for details).

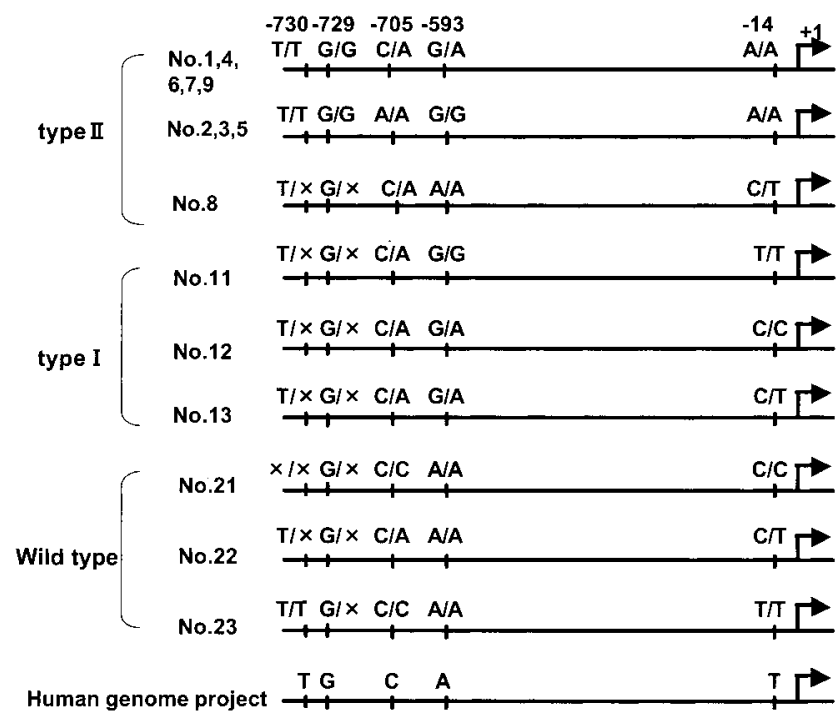

Fig. 3. SNPs in the Distal Promoter Regions of the Human FAT/CD36 Gene

Sequencing of 15 DNA samples ( 9 type II FAT/CD36 deficient, 3 type I deficient and 3 normal individuals) revealed 5 SNPs in the $1 \mathrm{~kb}$ region upstream of the distal transcriptional start site of the FAT/CD36 gene. The published human genome sequence is also shown and it coincides with one of those from normal individuals.

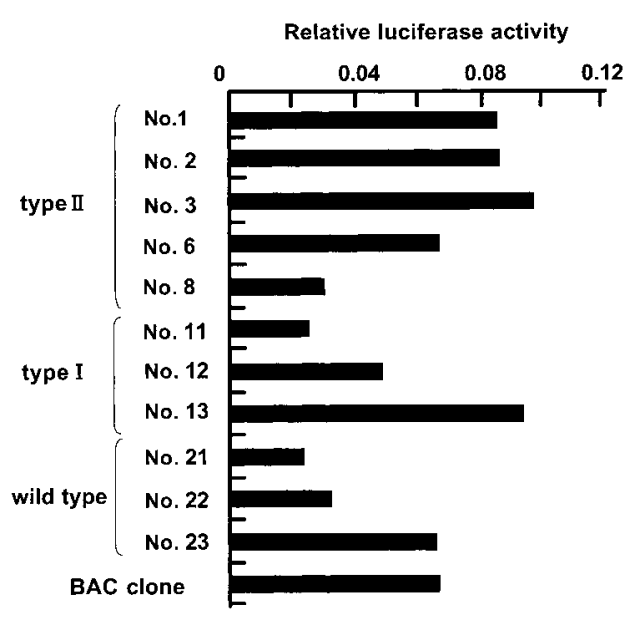

Fig. 4. Effects of the SNPs in the Distal Promoter Region on Expression of the Reporter Gene

Reporter assay with the distal FAT/CD36 gene promoter constructs constituting nucleotides -1500 to +1 relative to the transcriptional start site, and having various alleles for 5 SNPs. These constructs contained the cloned fragments and the combinations of heterozygous SNPs on the fragments were not determined. The experiment was independently repeated three times with similar results. 
active in this assay although reproducible variations in the activities even in the same group were observed probably because the cloned sequences contained either nucleotide at each heterogeneous SNP and different sequences could be obtained even from the DNA from the same subject. Furthermore, it should be noted that each promoter contains an additional 500 bp upstream sequence to that shown in Fig. 3 and may contain unidentified SNPs. Thus, each promoter sequence was somewhat different from the others except sequences \#1 and \#6 that showed similar activities. The SNPs at -14 and -593 did not diminish the promoter activity in this assay, suggesting that these identified SNPs are not directly linked to the cell type-specific deficiency of FAT/CD36 in platelets.

We found an association of the $-14 \mathrm{~A} / \mathrm{C}, \mathrm{T}$ polymorphism in the human FAT/CD36 gene promoter with the platelet-specific deficiency. However, this SNP does not seem to be directly associated with the transcriptional activity, although the possibility that the transcriptional regulation system in HepG2 cells is different from that in the progenitors of platelets and the in vivo inactive promoter is abnormally activated in HepG2 cells cannot be excluded. We speculate that mutations other than those identified in this study are responsible for the promoter inactivation and these mutations are strongly associated with the $-14 \mathrm{~A} / \mathrm{T}, \mathrm{C}$ SNP. It is clear that further studies with a larger number of subjects with the platelet-specific FAT/CD36 deficiency are necessary, and they are in progress in our laboratories in cooperation with others.

\section{REFERENCES}

1) Abumrad N. A., Maghrabi M. R., Amri E. Z., Lopez E., Grimaldi P. A., J. Biol. Chem., 268, 17665-17668 (1993).

2) Endemann G., Stanton L. W., Madden K. S., Bryant C. M., White R. T., Protter A. A., J. Biol. Chem., 268, 11811-11816 (1993).

3) Glatz J. F., Storch J., Curr. Opin. Lipidol., 12, 267—274 (2001).

4) Motojima K., Passilly P. P., Peters J. M., Gonzalez F. J., Latruffe N., J. Biol. Chem., 273, 16710-16714 (1998).

5) Nagy L., Tontonoz P., Alvalez J. G. A., Chen H., Evans R. M., Cell, 93, 229-240 (1998).

6) Griffin E., Re A., Hamel N., Fu C., Bush H., McCaffrey T., Asch A. S., Nat. Med., 7, 840-846 (2001).

7) Bonen A., Luiken J. J., Arumugam Y., Glatz J. F., Tandon N. N., J. Biol. Chem., 275, 14501-14508 (2000).

8) Aitman T. J., Glazier A. M., Wallace C. A., Cooper L. D., Norsworthy P. J., Wahid F. N., Al-Majali K. M., Trembling P. M., Mann C. J., Shoulders C. C., Graf D., St. Lezin E., W. Kurtz T., Kren V., Pravenec M., Ibrahimi A., Abumrad N. A., Stanton L. W., Scott J., Nat. Genet., 21, 76-83 (1999).

9) Febbraio M., Abumrad N. A., Hajjar D. P., Sharma K., Cheng W., Pearce S. F. A., Silverstein R. L., J. Biol. Chem., 274, 19055-19062 (1999).

10) Tanaka T., Nakata T., Oka T., Ogawa T., Okamoto F., Kusaka Y., Sohmiya K., Shimamoto K., Itakura K., J. Lipid Res., 42, 751-759 (2001).

11) Yamamoto N., Akamatsu N., Sakuraba H., Yamazaki H., Tanoue K., Blood, 83, 392-397 (1994).

12) Sato O., Kuriki C., Fukui Y., Motojima K., J. Biol. Chem., 277, 15703-15711 (2002).

13) Imai M., Tanaka T., Kintaka T., Ikemoto T., Shimizu A., Kitaura Y., Clin. Chim. Acta, 321, 97-106 (2002). 